\title{
Multi-dimensional Analysis, Information Mining and Numerical Modeling for Small Samples of Biomedical Microfluidic Droplets
}

\author{
Peiyuan $\mathrm{He}^{1, *}$, Liguo Zhang ${ }^{2}$ \\ ${ }^{1}$ School of life science, Zhengzhou Univ. 100 Science Avenue, Zhengzhou, P.R.China \\ ${ }^{2}$ Henan institute of pharmaceutical science, Zhengzhou University, Zhengzhou, P.R.China \\ *Corresponding author: hepeiyuan@live.com
}

Received August 25, 2014; Revised September 09, 2014; Accepted February 25, 2015

\begin{abstract}
Information mining has turned to be increasingly important in the modern digital world. The mining results lead to the essential advances in not only information technology, but general natural science, such as life science, mechanics, numerical analysis, calculation technology, etc. These innovations permitted for the manipulation of biomicrofluidic droplets at micrometer-scale. Through the acquisition of raw data and the multidimensional analysis, numerical modeling can possibly be established and the further optimization can be processed. This work was focused on the multi-dimensional analysis based on small samples of the biomicrofludic droplet generation. Then numerical modeling was used to describe the fabrication of microdroplets in complex circumstances. Both linear modeling and nonlinear optimization were performed while the artificial intelligence technology was applied. Although the linear model presented the rough descriptive capacities of microdroplets variation, non-linear optimization improved its descriptive properties. Artificial neural network was established to depict the microfluidic droplets and its accuracy was validated satisfactorily.
\end{abstract}

Keywords: information mining, data analysis, multi-dimensional analysis, biomicrofluidics, droplets, modeling

Cite This Article: Peiyuan He, and Liguo Zhang, "Multi-dimensional Analysis, Information Mining and Numerical Modeling for Small Samples of Biomedical Microfluidic Droplets." Biomedical Science and Engineering, vol. 3, no. 1 (2015): 1-3. doi: 10.12691/bse-3-1-1.

\section{Introduction}

The multidimensional analysis has been performed to evaluate the different variations in social life and scientific researches, giving birth to an effective tool to understand the nature of our observations. Taking a medical case for example, the medical effects on the patients and the necessary beliefs on their adherence to medication were assessed through clarifying the potentially complex interactions between different factors [1]. In cancer survivors, the good treatments, appropriate promotions and sufficiency for the accommodation can lead to attenuate the functional limitations at work [2]. Carcinogenesis biomarkers were evaluated through multidimensional analysis to overcome the deficiencies from single dimensional statistical analysis [3]. The application of multidimensional analysis facilitated the development in the fields of interest.

Biomicrofluidiscs, as an interdisciplinary subject, mainly combined the microfluidic technology and life science [4,5]. The micrometer-scale droplets with size homogeneity provided the same compartments to realize drug targeting, drug discovery [6] and food emulsions [7]. Biomicrofluidic droplets can be applied to test the heterogeneity in cytokine-secreting immune cells [8].
Microwell assays were performed to sort single cell based on images [9]. The mechanism of how a specific pharmaceutical was affected by a single cell was not well understood. The technical advancement limited the examination of little amounts of chemicals before microfluidic solutions appeared. Microfludics-based experimental techniques provided a way for single cell manipulation to analyze pharmacokinetics [10]. The advances in microfluidics brought about first-time, highresolution monitoring on the human immune systems. Microfluidic technology realized the diagnostic and prognostic examinations, while the quantitative general evaluation for immune system can be drawn [11]. It was reported that the combination of microwell technology and ELISA assay allowed for the possible goal-oriented diagnostics and drug susceptibility examinations [12].

The observed complexity made it not easy to describe the phenomena in biomicrofluidic environment [13]. Multi-dimensional analysis provided an effective way to depict different kinds of flow regimes in biomicrofluidics. Based on the analysis, the information mining allowed for the numerical modelling of the complex phenomena existing in the microfluidic channels.

The special shapes of microfluidic channels led to two different kinds of geometries, T-shape and flow-focusing shape [14]. The modeling on different droplet size evolution in these two geometries was distinguished due 
to the biomicrofluidic geometrical variations. Through including the force balance and geometrical effects, numerical modeling was established to describe the droplet flow under T-shaped microfluidic chips. The model can well explain the transition regimes of droplet formation [15]. However, flow-focusing shaped microfluidics is the other typical question for microdroplet flow. Small samples of microfluidic droplets were chosen as research object rather than large scale, for the large samples have been studied for a while. Furthermore, it is, to some extent, more difficult to establish an effective numerical model from small samples than at the moment when we had already known various situations from experimental observations. In this work, we at first presented experiments and multi-dimensional analyses on the small samples of microlfuidic droplets; secondly, the information mining was performed reasonably from the analyses to direct the establishments and optimization of numerical modelling; Thirdly, artificial neural network (ANN) was established to describe the microfluidic droplets and the validation procedures was performed to assess the accuracy of ANN model.

\section{Experiments and Multi-dimensional Analysis}

\subsection{Experiment Design}

The experiments were designed to test the possible combinations of flow rates, in order to clarify the role of each parameter. The central flow rate Qc was designed to include three levels, with a specific value each, as shown in Table 1; the lateral flow rates $\mathrm{Q}_{\mathrm{l}}$ consisted of three levels, each level with a particular value, shown in Table 2. Experiment conditions were distributed uniformly in the testing space, shown in Figure 1. This balanced design led to easy result comparison.

Table 1. Three $\mathbf{Q}_{\mathrm{c}}$ levels for corresponding values

\begin{tabular}{|c|c|c|c|}
\hline level & 1 & 2 & 3 \\
\hline value & 6.6 & 9.6 & 13.7 \\
\hline
\end{tabular}

Table 2. Three $Q_{l}$ levels for corresponding values

\begin{tabular}{|c|c|c|c|}
\hline Level & 1 & 2 & 3 \\
\hline Value & 10 & 15 & 20 \\
\hline
\end{tabular}

\subsection{Value Analysis}

Value analysis was undertaken to see the effects of $Q_{c}$ and $\mathrm{Q}_{\mathrm{l}}$, respectively. The results presented the relation between the droplet size $\mathrm{S}$ as function of $\mathrm{Q}_{c}$ and $\mathrm{Q}_{\mathrm{l}}$ in biomicrofluidics. S seemed to be on the direct proportion to $\mathrm{Q}_{c}$, while almost on the reverse proportion to $\mathrm{Q}_{\mathrm{l}}$. This experimental observations can be logically explained: for the increase of $Q_{c}$, the central viscous force became stronger, which took effects to give more time for droplet formation, permitting to enlarge droplet size; however, the augmentation of $\mathrm{Q}_{\mathrm{l}}$ led to enforce lateral shear stress on the central flow, allowing for the reduction of droplet sizes. We find the variation of droplet size for $\mathrm{Q}_{1}$ is 1.25 times as large as that of $\mathrm{Q}_{c}$. The $\mathrm{Q}_{\mathrm{l}}$ seemed to weigh more for droplet size variation in the process.

\subsection{Correlation Analysis}

The correlation analysis was performed between $\mathrm{Q}_{\mathrm{c}}$ and $\mathrm{Q}_{\mathrm{l}}$. $\mathrm{Q}_{\mathrm{c}}$ and $\mathrm{Q}_{\mathrm{l}}$ were found to be independent to each other, with $\mathrm{R}^{2} \approx 0$. This is a logic result for the flow rates adjustment were separately controlled between $\mathrm{Q}_{\mathrm{c}}$ and $\mathrm{Q}_{\mathrm{l}}$. This finding proposed a possibility to include these two flow rates into the mathematical model to express their linear behaviors for droplet generation.

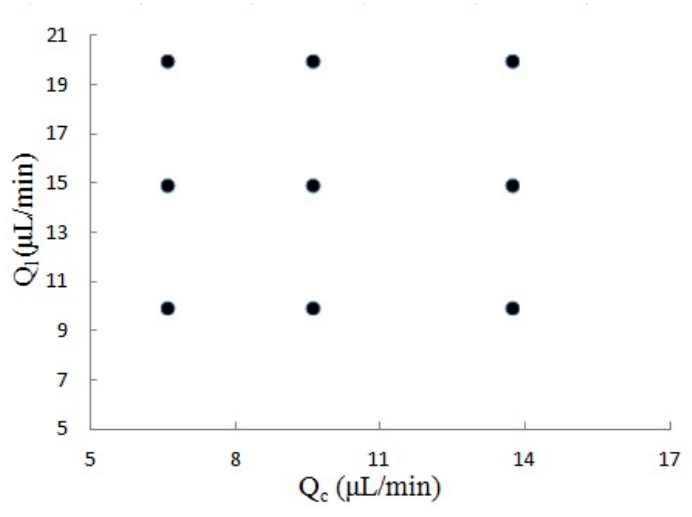

Figure 1. Scattering of $\mathrm{Q}_{c}$ and $\mathrm{Q}_{\mathrm{l}}$ in research space

\subsection{Modeling}

As shown in Equation 1, the linear model showed that as increase of $Q_{c}$ or decrease of $Q_{1}$. droplet sizes increased. The same phenomena were observed through different experiments. This result could be explained through mechanical viewpoint: In this biomicrofluidic environment, surface tension and viscous force take main effects. Surface tension between two flows tended to intercept flow elongation and facilitated the droplet formation, while the fluidic viscous force took more effects on keeping flow stable. In this study, the $\mathrm{Ca}$ $\sim O\left(10^{-1}\right)$, the surface tension overweighed the viscous force. This led to the formation of different droplets. Besides, the greater central flow rate $Q_{c}$ supported stronger viscous effects, permitting to extend the central flow much longer; while with augmentation of lateral flow rate $\mathrm{Q}_{1}$, the shear stress on the central flow becomes stronger, resulting in smaller droplets. The same phenomena were observed through different experiments.

$$
\mathrm{S}=24.5 \mathrm{Q}_{\mathrm{c}}-44.2 \mathrm{Q}_{1}+1011, R^{2}>0.85 .
$$

However, this linear model seemed to some extent to express droplet generation, but $R^{2}$ value was something short of effectiveness. The droplet generation was regarded as nonlinear process involving the interactions between pressures or flow-rates control, design of biomicrofuidic environment, and chemical interfacial compatibility. Then the nonlinear technique was used to optimize the modeling. The item $\left(\mathrm{Q}_{\mathrm{c}}{ }^{2}+\mathrm{Q}_{\mathrm{l}}{ }^{2}\right)^{0.5}$ was tested in the linear model to see the interaction between the two flow ratios. We added the nonlinear item $\left(\mathrm{Q}_{\mathrm{c}}{ }^{2}+\mathrm{Q}_{1}{ }^{2}\right)^{0.5}$ to the model to involve the interaction effects.

\subsection{Nonlinear Optimization}

The modeling for droplet generation was optimized with the inclusion of flow rates item $\left(\mathrm{Q}_{\mathrm{c}}{ }^{2}+\mathrm{Q}_{\mathrm{l}}{ }^{2}\right)^{0.5}$, as presented in Equation. 2. The improved nonlinear model could explain well the droplet size variation. 


$$
S=-49 Q c-150 \mathrm{Q}_{1}+129\left(\mathrm{Q}_{\mathrm{c}}{ }^{2}+\mathrm{Q}_{1}{ }^{2}\right)^{0.5}+975, R^{2}>0.9 .
$$

This nonlinear model showed that as the increase of $\mathrm{Q}_{\mathrm{c}}$ or $\mathrm{Q}_{\mathrm{l}}$, the droplet sizes decreased; besides, with the increase of flow rates item $\left(\mathrm{Q}_{c}{ }^{2}+\mathrm{Q}_{1}{ }^{2}\right)^{0.5}$, droplet sizes varied towards the opposite direction. For $\mathrm{Q}_{1}$, droplet sizes varying as the same results as linear model; For $\mathrm{Q}_{\mathrm{c}}$, however, droplet sizes varied differently comparing with linear model. This difference consisted in the inclusion of nonlinear item $\left(\mathrm{Q}_{\mathrm{c}}{ }^{2}+\mathrm{Q}_{\mathrm{l}}{ }^{2}\right)^{0.5}$, showing that the interaction effects could take effects on each item at the model. The addition of nonlinear item improved the model quality but also expressed the descriptive complexity for the droplet variation. The conclusion deduced from this model coincided well with the experimental observations.

\section{Neural Network Modeling and Verification}

The Neural Network Modeling (NNM) for droplet size variation was performed. The $\mathrm{Q}_{c}$ and $\mathrm{Q}_{1}$ were contained in the input layer, while the response in this non-linear model represented the droplet size. The theoretical analysis was performed to compare the values extracted from experiments with the theoretical ones calculated through neural networks modeling. The validated results were presented in the Figure 2. These predicted values fell in the vicinity of the experimental curve, demonstrating the effectiveness of this modeling based on the artificial neural network technology.

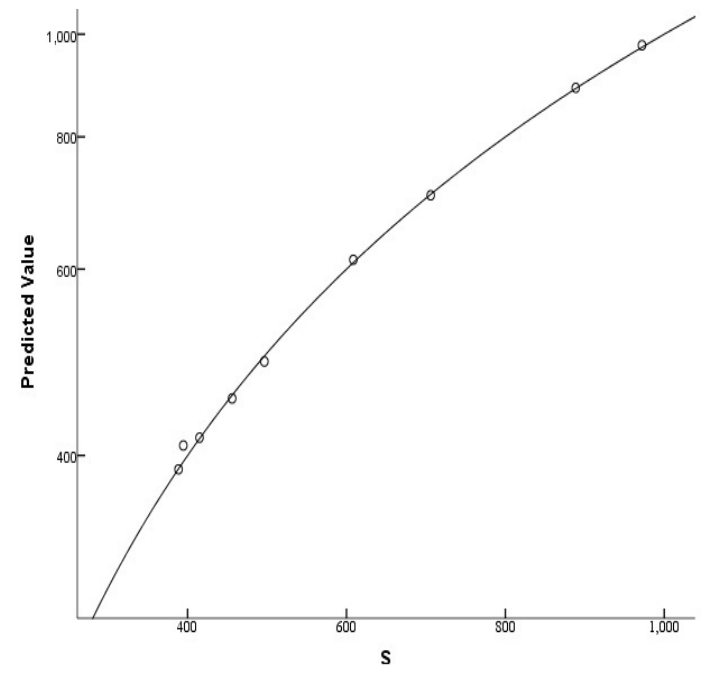

Figure 2. Verification of Neural Network Modeling (NNM)

\section{Conclusion}

In this work, it is demonstrated that multidimensional analyses were proved to be an effective way to understand the inside mechanism in biomicrofludic droplet generation. Especially, the role for two independent factors $\mathrm{Q}_{\mathrm{c}}$ and $\mathrm{Q}_{\mathrm{l}}$ was examined. The $\mathrm{Q}_{\mathrm{c}}$ was found to weigh less than $\mathrm{Q}_{\mathrm{l}}$ in droplet variation. The information mining from small samples facilitated the establishment of the numerical models. The linear model could present the rough descriptive effectiveness. The non-linear optimizations ameliorated the descriptive satisfaction of numerical model. ANN technology was introduced to simulate the droplet variations. It was found that ANN brought satisfactory accuracy to the droplet variation through the validation procedures.

\section{Acknowledgment}

This work was supported by the Key Program for Science and Technology Development of education department of Henan province(No. 14A180019), and by the natural science foundation of Henan province (No.142300410459).

\section{References}

[1] L. Alison Phillips, Michael A. Diefenbach, Ian M. Kronish, Rennie M. Negron, Carol R. Horowitz, "The Necessity-Concerns Framework: a Multidimensional Theory Benefits from Multidimensional Analysis" Annals of Behavioral Medicine, Volume 48, pp 7, 2014.

[2] Michal C. Moskowitz, Briana L. Todd, Rusan Chen, Michael Feuerstein, "Function and friction at work: a multidimensional analysis of work outcomes in cancer survivors” Journal of Cancer Survivorship Volume 8, pp 173, 2014.

[3] Marisa Mariani, Shiquan He, Mark McHugh, Mirko Andreoli, Deep Pandya, Steven Sieber, Zheyang Wu, Paul Fiedler, Shohreh Shahabi, Cristiano Ferlini, "Integrated Multidimensional Analysis Is Required for Accurate Prognostic Biomarkers in Colorectal Cancer” Plos one, Volume 9, pp 101065, 2014.

[4] Peiyuan He, Xue Lexun, Qi Yuanming, Zhang Liguo, Lu Yumin, Zhang erliang: "Numerical Information Retrieval and Modeling for Small Droplet Samples.” WIT transactions on engineering science. Volume 86, pp 371-376, 2014.

[5] Peiyuan He, D.Barthes-Biesel, E. Leclerc. "Flow of two immiscible liquids in Y-shaped microfluidic systems: effect of geometry.” Microfluidics and nanofluidics. Volume 9, pp 293, 2010.

[6] Petra S. Dittrich and Andreas Manz, "Lab-on-a-chip: microfluidics in drug discovery,” Nature Reviews Drug Discovery, Volume 5, pp 210, 2006.

[7] George A. van Aken, "Relating Food Emulsion Structure and Composition to the Way It Is Processed in the Gastrointestinal Tract and Physiological Responses: What Are the Opportunities?” Food Biophysics, Volume 5, pp. 258, 2010.

[8] Venkatachalam Chokkalingam, Jurjen Tel, Florian Wimmers, Xin Liu, Sergey Semenov, Julian Thiele, Carl G. Figdorb and Wilhelm T. S. Huck "Probing cellular heterogeneity in cytokine-secreting immune cells using droplet-based microfluidics” Lab on Chip, Volume 13, pp 4740, 2013.

[9] Tao Sun, Joseph Kovac, and Joel Voldman, "Image-Based SingleCell Sorting via Dual-Photopolymerized Microwell Arrays.” Anal. Chem., Volume 86, pp 977, 2014.

[10] Yong Luo, Chang Liu, Yueyang Qu and Ning Fang. "Towards single-cell analysis for pharmacokinetics.” Bioanalysis. Vol. 4, pp 453, 2012.

[11] Shai S. Shen-Orr, "Challenges and Promise for the Development of Human Immune Monitoring.” Rambam Maimonides Med J. Volume 3, pp 0023, 2012.

[12] Yen H. Nguyen, Xin Ma, and Lidong Qin, "Rapid identification and drug susceptibility screening of ESAT-6 secreting Mycobacteria by a NanoELIwell assay.” Science, Volume 2, pp 635, 2012.

[13] Zhang Liguo, Xue Lexun, He Peiyuan, Qi Yuanming, Lu Yumin, "Intelligent numerical manipulation of micrometer-scale emulsions using polymer confinement.” Advanced Materials Research, Volume813, pp 431, 2013.

[14] He Peiyuan, Xue Lexun, Zhang Liguo, Qi Yuanming, Lu Yumin, “Artificial Neural network(ANN)-BasedNonlinear Optimization of Modeling on Biomicrofluidic Vesicles Generation.” 9th International Conference on Natural Computation, IEEE, 2013.

[15] Tomasz Glawdel, Caglar Elbuken, and Carolyn L. Ren, "Droplet formation in microfluidic T-junction generators operating in the transitional regime.” Phys Rev E Stat Nonlin Soft Matter Phys. Volume 85, pp 016322, 2012. 